\title{
GROTHENDIECK-LIDSKII TRACE FORMULA FOR MIXED-NORM AND VARIABLE LEBESGUE SPACES
}

\author{
JULIO DELGADO, MICHAEL RUZHANSKY, AND BAOXIANG WANG \\ To the memory of Yuri Safarov
}

\begin{abstract}
In this note we present the metric approximation property for weighted mixed-norm $L_{w}^{\left(p_{1}, \ldots, p_{n}\right)}$ and variable exponent Lebesgue type spaces. As a consequence, this also implies the same property for modulation and Wiener-Amalgam spaces. We then characterise nuclear operators on such spaces and state the corresponding Grothendieck-Lidskii trace formulae. We apply the obtained results to derive criteria for nuclearity and trace formulae for periodic operators on $\mathbb{R}^{n}$ and functions of the harmonic oscillator in terms of global symbols.
\end{abstract}

\section{INTRODUCTION}

The famous Lidskii formula Lid59] states the equality between the operator trace of trace-class operators in Hilbert spaces and the sum of their eigenvalues. Similar properties can be established in Banach spaces using Grothendieck's theory of nuclear operators. The basic ingredient for such an approach is the approximation property of the Banach space under consideration. In this paper we describe the approximation properties and the subsequent Grothendieck-Lidskii formulae

Date: April 4, 2016.

2010 Mathematics Subject Classification. Primary 46B26, 47B38; Secondary 47G10, 47B06, 42B35.

Key words and phrases. Mixed-norm Lebesgue spaces, modulation spaces, Wiener amalgam spaces, nuclearity, trace formulae, harmonic oscillator.

The first author was supported by the Leverhulme Research Grant RPG-201402. The second author was supported by the EPSRC Grant EP/K039407/1. No new data was collected or generated during the course of the research. The authors have been also supported by the Sino-UK research project by the British Council China and the China Scholarship Council, and by EPSRC Mathematics Platform grant EP/I019111/1. 
for the traces of operators in weighted mixed-norm and in variable exponent Lebesgue spaces.

The approximation property is one of the fundamental properties of the 'geometry' of Banach spaces. A particular importance of this property from the Grothendieck's viewpoint is that once a Banach space is known to have it, the trace can be defined and consequently the Fredholm's determinant leading to numerous further developments. Thus, the topic finds itself closely related to a wide range of analysis: spectral analysis, operator theory, functional analysis, harmonic analysis, PDEs. In particular, nuclearity properties of a given operator allow to obtain information on the distribution eigenvalues through the use of Grothendieck, König, Maurey, Retherford and Johnson inequalities which can be seen as an extension of the classical Weyl inequality relating sums of eigenvalues and Schatten-von Neumann norms.

In the paper we present the metric approximation property for three scales of spaces that are of importance in a broad range of mathematical subjects. First, the mixed Lebesgue spaces are a basic tool for harmonic analysis and evolutions PDEs (Strichartz estimates). The approximation property of such spaces ([DRW]) gives rise to an introduction of spectral methods (following Grothendieck) to a variety of questions of harmonic analysis and PDEs. A part of the paper is also devoted to the development of these ideas. Second, Wiener amalgam spaces are a central object of the time-frequency analysis, another area with links to several mathematical subjects as well as its applications. Also, the approximation property in the scale of modulation spaces gives rise to the introduction of spectral analysis to PDEs of very different type - these spaces become more and more effective (compared to Besov spaces) in many types of PDEs including such PDEs as the Navier-Stokes equation, e.g. Iwa10. Finally, we discuss (following [DR] ) the validity of the metric approximation property for the variable exponent Lebesgue spaces. In all the considered spaces a characterisation of nuclear operators is established and the corresponding Grothendieck-Lidskii formulae for the trace are derived. In the case of variable exponent Lebesgue spaces on the torus we present sufficient conditions to ensure the nuclearity of an operator of the form $\alpha(x)(I-\Delta)^{-\frac{\tau}{2}}$, where $\alpha$ is a suitable function.

Let $\mathcal{B}_{1}, \mathcal{B}_{2}$ be Banach spaces and let $0<r \leq 1$. A linear operator $T$ from $\mathcal{B}_{1}$ into $\mathcal{B}_{2}$ is called $r$-nuclear if there exist sequences $\left(x_{n}^{\prime}\right)$ in $\mathcal{B}_{1}^{\prime}$ 
and $\left(y_{n}\right)$ in $\mathcal{B}_{2}$ so that $T x=\sum_{n=1}^{\infty}\left\langle x, x_{n}^{\prime}\right\rangle y_{n}$ and $\sum_{n=1}^{\infty}\left\|x_{n}^{\prime}\right\|_{\mathcal{B}_{1}^{\prime}}^{r}\left\|y_{n}\right\|_{\mathcal{B}_{2}}^{r}<$ $\infty$. When $r=1$ the notion of nuclear operators agrees with the one of trace class operators in the setting of Hilbert spaces $\left(\mathcal{B}_{1}=\mathcal{B}_{2}=H\right)$. Grothendieck Gro55] proved that the trace $\operatorname{Tr}(T)$ is well defined for all nuclear operators if and only if the Banach space $\mathcal{B}$ has the approximation property, i.e. for every compact set $K$ in $\mathcal{B}$ and for every $\epsilon>0$, there exists $F \in \mathcal{F}(\mathcal{B})$ such that $\|x-F x\|<\epsilon, \quad$ for all $x \in K$, where $\mathcal{F}(\mathcal{B})$ denotes the space of finite rank bounded linear operators on $\mathcal{B}$. If in the definition above the operator $F$ satisfies $\|F\| \leq 1$ one says that $\mathcal{B}$ possesses the metric approximation property. In Gro55 Grothendieck proved that if $T$ is $\frac{2}{3}$-nuclear from $\mathcal{B}$ into $\mathcal{B}$, then its trace agrees with the sum of eigenvalues. The first example of a Banach space without the approximation property appeared in [Enf73]. In [Sza81] Szankowski proved that $B(H)$ does not have the approximation property. More recently, these properties have been intensively also investigated in different scales of function spaces, see e.g. [ACPP05, [JS12, [LLO10.

\section{Mixed-Normed $L^{p}$, Modulation and Wiener-Amalgam SPACES}

The modulation spaces have been intensively investigated in the last decades. Modulation spaces start finding numerous applications in various problems in linear and nonlinear partial differential equations, see RSW12 for a recent survey. For a suitable weight $w$ on $\mathbb{R}^{2 d}$, $1 \leq p, q<\infty$ and a window $g \in \mathcal{S}\left(\mathbb{R}^{d}\right)$ the modulation space $\mathcal{M}_{w}^{p, q}\left(\mathbb{R}^{d}\right)$ consists of the temperate distributions $f \in \mathcal{S}^{\prime}\left(\mathbb{R}^{d}\right)$ such that

$$
\begin{gathered}
\|f\|_{\mathcal{M}_{w}^{p, q}}:=\left\|V_{g} f\right\|_{L_{w}^{p, q}}:= \\
\left(\int_{\mathbb{R}^{d}}\left(\int_{\mathbb{R}^{d}}\left|V_{g} f(x, \xi)\right|^{p} w(x, \xi)^{p} d x\right)^{\frac{q}{p}} d \xi\right)^{\frac{1}{q}}<\infty
\end{gathered}
$$

where $V_{g} f(x, \xi)$ denotes the short-time Fourier transform of $f$ with respect to $g$ at the point $(x, \xi)$. The modulation space $\mathcal{M}_{w}^{p, q}\left(\mathbb{R}^{d}\right)$ endowed with the above norm becomes a Banach space, independent of $g \neq 0$. A weight function is a non-negative, locally integrable function on $\mathbb{R}^{2 d}$. A weight function $v$ on $\mathbb{R}^{2 d}$ is called submultiplicative, if

$$
v(x+y) \leq v(x) v(y) \text { for all } x, y \in \mathbb{R}^{2 d} .
$$


A weight function $w$ on $\mathbb{R}^{2 d}$ is v-moderate, if

$$
w(x+y) \leq v(x) w(y) \text { for all } x, y \in \mathbb{R}^{2 d} .
$$

In particular the weights of polynomial type play an important role. They are of the form

$$
v_{s}(x, \xi)=\left(1+|x|^{2}+|\xi|^{2}\right)^{s / 2} .
$$

The $v_{s}$-moderated weights (for some $s$ ) are called polynomially moderated.

We now recall the definition of weighted mixed-norm $L^{p}$ spaces. Let $\left(\Omega_{i}, S_{i}, \mu_{i}\right)$, for $i=1, \ldots, n$, be given $\sigma$-finite measure spaces. We write $x=\left(x_{1}, \ldots, x_{n}\right)$, and let $P=\left(p_{1}, \ldots, p_{n}\right)$ a given $n$-tuple with $1 \leq p_{i}<\infty$. We say that $1 \leq P<\infty$ if $1 \leq p_{i}<\infty$ for all $i=1, \ldots, n$. Let $w$ be a strictly positive measurable function. The norm $\|\cdot\|_{L_{w}^{P}}$ of a measurable function $f\left(x_{1}, \ldots, x_{n}\right)$ on the corresponding product measure space is defined by

$$
\begin{gathered}
\|f\|_{L_{w}^{P}}:=\left(\int _ { \Omega _ { n } } \cdots \left(\int_{\Omega_{2}}\right.\right. \\
\left.\left.\left(\int_{\Omega_{1}}|f(x)|^{p_{1}} w(x) d \mu_{1}\left(x_{1}\right)\right)^{\frac{p_{2}}{p_{1}}} d \mu_{2}\left(x_{2}\right)\right)^{\frac{p_{3}}{p_{2}}} \cdots d \mu_{n}\left(x_{n}\right)\right)^{\frac{1}{p_{n}}} .
\end{gathered}
$$

$L_{w}^{P}$-spaces endowed with the $\|\cdot\|_{L_{w}^{P} \text {-norm become Banach spaces and }}$ the dual $\left(L_{w}^{P}\right)^{\prime}$ of $L_{w}^{P}$ is $L_{w^{-1}}^{P^{\prime}}$, where $P^{\prime}=\left(p_{1}^{\prime}, \ldots, p_{n}^{\prime}\right)$. In view of our application to the modulation spaces $\mathcal{M}_{w}^{p, q}$ we will consider in particular the case of the index of the form $(P, Q)=\left(p_{1}, \ldots, p_{d}, q_{1}, \ldots, q_{d}\right)$ where $p_{i}=p, q_{i}=q$ and $\Omega_{i}=\mathbb{R}$ endowed with the Lebesgue measure. In this case the weight is taken in the form $w=w(x, \xi)$ where $x \in \mathbb{R}^{d}, \xi \in \mathbb{R}^{d}$. In the rest of this section we will assume that the weights $w$ satisfy the following condition for all $x \in \Omega$ :

$$
w\left(x_{1}, \ldots, x_{n}\right) \leq w_{1}\left(x_{1}\right) \cdots w_{n}\left(x_{n}\right),
$$

where $w_{j}$ is a weight on $\Omega_{j}$ (i.e. a strictly positive locally integrable function). In particular, the condition holds for polynomially moderate weights on $\mathbb{R}^{n}$ satisfying for a suitable $n$-tuple $\left(\beta_{1}, \ldots, \beta_{n}\right)$ the condition

$$
w\left(x_{1}, \ldots, x_{n}\right) \leq\left\langle x_{1}\right\rangle^{\beta_{1}} \cdots\left\langle x_{n}\right\rangle^{\beta_{n}},
$$

where $\left\langle x_{j}\right\rangle=1+\left|x_{j}\right|$. The following theorem was established in [DRW]. 
Theorem 2.1. The weighted mixed-norm spaces $L_{w}^{P}=L_{w}^{\left(p_{1}, \ldots, p_{n}\right)}$ with $w$ satisfying (5) have the metric approximation property.

Let us recall now the definition of the Wiener amalgam spaces $\mathcal{W}_{w}^{p, q}\left(\mathbb{R}^{d}\right)$. There are several definitions possible for the spaces $\mathcal{W}_{w}^{p, q}$, in particular involving the short-time Fourier transform similarly to the definition of the modulation spaces in (1). To make an analogy with modulation spaces, we can reformulate their definition (1) in terms of the mixed-normed Lebesgue spaces, by saying that

$$
f \in \mathcal{M}_{w}^{p, q}\left(\mathbb{R}^{d}\right) \text { if and only if } V_{g} f \cdot w \in L^{(p, q)}\left(\mathbb{R}^{d} \times \mathbb{R}^{d}\right) .
$$

Now, for a function $F \in L_{\text {loc }}^{1}\left(\mathbb{R}^{2 d}\right)$, we denote $\mathcal{R} F(x, \xi):=F(\xi, x)$. Then we can define

$$
f \in \mathcal{W}_{w}^{p, q}\left(\mathbb{R}^{d}\right) \text { if and only if } \mathcal{R}\left(V_{g} f \cdot w\right) \in L^{(q, p)}\left(\mathbb{R}^{d} \times \mathbb{R}^{d}\right) .
$$

However, for our purposes the following description through the Fourier transform will be more practical. For a review of different definitions we refer to [RSTT11]. So, in what follows, we will always assume that the weights in modulation and Wiener amalgam spaces are submultiplicative and polynomially moderate (but we do not need to assume this when talking about weighted mixed-norm $L^{P}$-spaces) as in (2)(4). Then, because of the identity

$$
\left|V_{g} f(x, \xi)\right|=(2 \pi)^{-d}\left|V_{\widehat{g}} \widehat{f}(\xi,-x)\right|,
$$

the Wiener amalgam space $\mathcal{W}_{w}^{p, q}$ and the modulation spaces are related through the Fourier transform by the formula

$$
\|f\|_{\mathcal{W}_{w}^{p, q}} \simeq\|\widehat{f}\|_{\mathcal{M}_{w_{0}}^{q, p}}
$$

where $w(x, \xi)=w_{0}(\xi,-x)$. As a consequence of Theorem 2.1 we now immediately obtain:

Corollary 2.2. Let $1 \leq p, q<\infty$, and $w$ a submiltiplicative polynomially moderate weight. Then $\mathcal{M}_{w}^{p, q}$ has the metric approximation property. Consequently, also the Wiener amalgam space $\mathcal{W}_{w}^{p, q}$ has the metric approximation property.

It was observed by Feichtinger and Gröchenig [FG89] that the metric approximation property could be alternatively established for the corresponding sequence spaces using appropriate atomic decompositions. Thus, Corollary 2.2 implies the metric approximation property for the sequence spaces arising through the atomic decompositions of $\mathcal{M}_{w}^{p, q}$ and $\mathcal{W}_{w}^{p, q}$. 
In order to formulate a characterisation of $r$-nuclear operators between weighted mixed-norm spaces we will consider $1 \leq P, Q<\infty$. The multi-index $P$ will be associated to the measures $\mu_{i}(i=1, \ldots, l)$ and $Q$ will correspond to the measures $\nu_{j}(j=1, \ldots, m)$. We will also denote $\mu:=\mu_{1} \otimes \cdots \otimes \mu_{l}$ and $\nu:=\nu_{1} \otimes \cdots \otimes \nu_{m}$ the corresponding product measures on the product spaces $\Omega=\prod_{i=1}^{l} \Omega_{i}, \Xi=\prod_{j=1}^{m} \Xi_{j}$. For a weight $w$ we will denote $w_{P}(\Omega):=\left\|1_{\Omega}\right\|_{L_{w}^{P}(\mu)}$. The additional property (5) will be only required for the formulation of trace relations.

Definition 2.3. Let $\left(\Omega_{i}, \mathcal{M}_{i}, \mu_{i}\right)(i=1, \ldots, l)$ be measure spaces and $\mu:=\mu_{1} \otimes \cdots \otimes \mu_{l}$ the corresponding product measure on $\Omega=\prod_{i=1}^{l} \Omega_{i}$. We will also call $\Lambda \in \mathcal{M}:=\bigotimes_{i=1}^{l} \mathcal{M}_{i}$ a box if it is of the form $\Lambda=\prod_{i=1}^{l} \Lambda_{i}$. For a measure $\mu$, a weight $w$ on $\Omega$ and a multi-index $P$ we will say that the triple $(\mu, w, P)$ is $\sigma$-finite if there exists a family of disjoint boxes $\Omega^{k}$ such that $\mu\left(\Omega^{k}\right)<\infty, \bigcup_{k=1}^{\infty} \Omega^{k}=\Omega$ and

$$
w_{P}\left(\Omega^{k}\right)=\left\|1_{\Omega^{k}}\right\|_{L_{w}^{P}(\mu)}<\infty .
$$

We can now give a characterisation of $r$-nuclear operators on weighted mixed-norm spaces and a trace formula.

Theorem 2.4. Let $0<r \leq 1$. Let $\left(\Omega_{i}, \mathcal{M}_{i}, \mu_{i}\right)(i=1, \ldots, l)$, $\left(\Xi_{j}, \mathcal{M}_{j}^{\prime}, \nu_{j}\right)(j=1, \ldots, m)$ be measure spaces. Let $1 \leq P, Q<\infty$. Let $w, \widetilde{w}$ be weights on $\Omega, \Xi$ respectively such that the triples $(\mu, w, P)$, $\left(\nu, \widetilde{w}^{-1}, Q^{\prime}\right)$ are $\sigma$-finite. Then $T$ is $r$-nuclear operator from $L_{w}^{P}(\mu)$ into $L_{\widetilde{w}}^{Q}(\nu)$ if and only if there exist a sequence $\left(g_{n}\right)$ in $L_{\widetilde{w}}^{Q}(\nu)$, and a sequence $\left(h_{n}\right)$ in $L_{w^{-1}}^{P^{\prime}}(\mu)$ such that $\sum_{n=1}^{\infty}\left\|g_{n}\right\|_{L_{\tilde{w}}^{Q}(\nu)}^{r}\left\|h_{n}\right\|_{L_{w^{-1}}^{P^{\prime}}(\mu)}^{r}<\infty$, and such that for all $f \in L_{w}^{P}(\mu)$

$$
T f(x)=\int_{\Omega}\left(\sum_{n=1}^{\infty} g_{n}(x) h_{n}(y)\right) f(y) d \mu(y), \text { for a.e } x \text {. }
$$

Moreover, if $w=\widetilde{w}$ satisfies (5), $\mu=\nu, P=Q$ and $T$ is $r$-nuclear in $\mathcal{L}\left(L_{w}^{P}(\mu)\right)$ with $r \leq \frac{2}{3}$, then

$$
\operatorname{Tr}(T)=\sum_{j=1}^{\infty} \lambda_{j}
$$


where $\lambda_{j}(j=1,2, \ldots)$ are the eigenvalues of $T$ with multiplicities taken into account, and $\operatorname{Tr}(T)=\sum_{j=1}^{\infty}\left\langle u_{j}, v_{j}\right\rangle$.

Analogous characterisations of $r$-nuclear operators can be obtained in the case of modulation spaces and Wiener-Amalgam spaces (cf. [DRW]). We now formulate an application in the case of modulation spaces to the study of functions of the harmonic oscillator $A=-\Delta+$ $|x|^{2}$ on $\mathbb{R}^{d}$, defined by

$$
F\left(-\Delta+|x|^{2}\right) \phi_{j}=F\left(\lambda_{j}\right) \phi_{j}, \quad j=1,2, \ldots,
$$

where $\lambda_{j}$ 's are the eigenvalues of $A$. We have:

Theorem 2.5. Let $0<r \leq 1, s \in \mathbb{R}$ and $1 \leq p, q<\infty$. The operator $F\left(-\Delta+|x|^{2}\right)$ is r-nuclear on $\mathcal{M}_{s}^{p, q}\left(\mathbb{R}^{d}\right)$ provided that

$$
\sum_{j=1}^{\infty}\left|F\left(\lambda_{j}\right)\right|^{r}\left\|\phi_{j}\right\|_{\mathcal{M}_{s}^{p, q}}^{r}\left\|\phi_{j}\right\|_{\mathcal{M}_{-s}^{p^{\prime}, q^{\prime}}}^{r}<\infty .
$$

Moreover, if (11) holds with $r=1$, we have the trace formula

$$
\operatorname{Tr} F\left(-\Delta+|x|^{2}\right)=\sum_{j=1}^{\infty} F\left(\lambda_{j}\right),
$$

with the absolutely convergent series.

\section{VARIABle EXPONENT LEBESGUe SPACES}

The variable exponent Lebesgue spaces are a generalisation of the classical Lebesgue spaces, replacing the constant exponent $p$ by a variable exponent function $p(x)$. The development of the analysis of many problems on those spaces has been of great interest in the last decades as has been exhibited in the recent books DHHR11, CUFRW14. and the literature therein. We now recall the definition of variable exponent Lebesgue spaces and refer the reader to [DHHR11] for the basic properties of such spaces. Let $(\Omega, \mathcal{M}, \mu)$ be a $\sigma$-finite, complete measure space. We define $\mathcal{P}(\Omega, \mu)$ to be the set of all $\mu$-measurable functions $p: \Omega \rightarrow[1, \infty]$. The functions in $\mathcal{P}(\Omega, \mu)$ are called variable exponents on $\Omega$. We define $p^{+}=p_{\Omega}^{+}:=\operatorname{ess}_{\sup _{x \in \Omega}} p(x), \quad p^{-}=p_{\Omega}^{-}:=$ $\operatorname{essinf}_{x \in \Omega} p(x)$. If $p^{+}<\infty$, then $p$ is called a bounded variable exponent. If $f: \Omega \rightarrow \mathbb{R}$ is a measurable function we define the modular 
associated with $p=p(\cdot)$ by

$$
\rho_{p(\cdot)}(f):=\int_{\Omega}|f(x)|^{p(x)} d \mu(x),
$$

and $\|f\|_{L^{p(\cdot)(\mu)}}:=\inf \left\{\lambda>0: \rho_{p(\cdot)}(f / \lambda) \leq 1\right\}$. The resulting spaces $L^{p(\cdot)}(\mu)$ of measurable functions such that $\|f\|_{L^{p(\cdot)}(\mu)}<\infty$ are Banach spaces and enjoy many properties similar to the classical Lebesgue $L^{p}$ spaces. If the variable exponent $p(\cdot)$ is bounded the space $L^{p(\cdot)}(\mu)$ is separable and if we denote by $p^{\prime}(\cdot)$ the variable exponent defined pointwise by $\frac{1}{p(x)}+\frac{1}{p^{\prime}(x)}=1$, then $\left(L^{p(\cdot)}(\mu)\right)^{\prime}=L^{p^{\prime}(\cdot)}(\mu)$. Moreover, if $1<p^{-} \leq p^{+}<\infty$ the space $L^{p(\cdot)}(\mu)$ is reflexive. For the study of the approximation property we will restrict to consider bounded variable exponents due to the density of the simple functions in $L^{p(\cdot)}$ in that case. We can now state the metric approximation property which was proved in [DR]:

Theorem 3.1. Let $p \in \mathcal{P}(\Omega, \mu)$ be a bounded variable exponent. Then, the variable exponent Lebesgue space $L^{p(\cdot)}(\mu)$ has the metric approximation property.

We are now ready to give a characterisation of $r$-nuclear operators for variable exponent spaces.

Theorem 3.2. Let $(\Omega, \mathcal{M}, \mu)$ and $\left(\Xi, \mathcal{M}^{\prime}, \nu\right)$ be $\sigma$-finite complete measure spaces. Let $0<r \leq 1$. Then $T$ is $r$-nuclear operator from $L^{p(\cdot)}(\mu)$ into $L^{q(\cdot)}(\nu)$ if and only if there exist a sequence $\left(g_{n}\right)$ in $L^{q(\cdot)}(\nu)$, and a sequence $\left(h_{n}\right)$ in $L^{p^{\prime}(\cdot)}(\mu)$ such that $\sum_{n=1}^{\infty}\left\|g_{n}\right\|_{L^{q(\cdot)}(\nu)}^{r}\left\|h_{n}\right\|_{L^{p^{\prime}(\cdot)}(\mu)}^{r}<\infty$, and such that for all $f \in L^{p(\cdot)}(\mu)$ we have

$$
T f(x)=\int_{\Omega}\left(\sum_{n=1}^{\infty} g_{n}(x) h_{n}(y)\right) f(y) d \mu(y) \text {, for a.e } x \text {. }
$$

Moreover, if $\Omega=\Xi, \mu=\nu, p(\cdot)=q(\cdot), p^{+}<\infty$, and $T$ is $r$-nuclear in $\mathcal{L}\left(L^{p(\cdot)}(\mu)\right)$ with $r \leq \frac{2}{3}$, then

$$
\operatorname{Tr}(T)=\sum_{j=1}^{\infty} \lambda_{j}
$$


where $\lambda_{j}(j=1,2, \ldots)$ are the eigenvalues of $T$ on $L^{p(\cdot)}(\mu)$ with multiplicities taken into account, and

$$
\operatorname{Tr}(T)=\sum_{n=1}^{\infty}\left\langle g_{n}, h_{n}\right\rangle=\int_{\Omega} \sum_{n=1}^{\infty} g_{n}(x) h_{n}(x) d \mu .
$$

We denote the $n$-dimensional torus by $\mathbb{T}^{n}=\mathbb{R}^{n} / \mathbb{Z}^{n}$. Its unitary dual can be described as $\widehat{\mathbb{T}^{n}} \simeq \mathbb{Z}^{n}$, and the collection $\left\{\xi_{k}(x)=e^{2 \pi i x \cdot k}\right\}_{k \in \mathbb{Z}^{n}}$ is an orthonormal basis of $L^{2}\left(\mathbb{T}^{n}\right)$. A corresponding operator is associated to a symbol $\sigma(x, \xi)$ which will be called a periodic pseudodifferential operator or the operator given by the toroidal quantization:

$$
T_{\sigma} f(x)=\sum_{\xi \in \mathbb{Z}^{n}} e^{2 \pi i x \cdot \xi} \sigma(x, \xi)\left(\mathcal{F}_{\mathbb{T}^{n}} f\right)(\xi)
$$

which can also be written as

$$
T_{\sigma} f(x)=\sum_{\xi \in \mathbb{Z}^{n}} \int_{\mathbb{T}^{n}} e^{2 \pi i(x-y) \cdot \xi} \sigma(x, \xi) f(y) d y .
$$

We refer to [RT10b] for an extensive analysis of such toroidal quantization, and to [RT10a] for the toroidal background analysis. In the rest of this section we will consider $\mathbb{T}^{n}$ endowed with the Borel $\sigma$ algebra and the Lebesgue measure so that we will just write $\mathcal{P}\left(\mathbb{T}^{n}\right)$ to denote the corresponding class of variable exponents. Given a measurable function $\alpha$ on $\mathbb{T}^{n}$, we take the symbols $\alpha(x)$ and $\sigma(\xi)$, the corresponding multiplication is the operator denoted by $\alpha T_{\sigma}$ given by $\alpha T_{\sigma} f=\alpha \sigma(D) f$ on $\mathbb{T}^{n}$.

Corollary 3.3. Let $p(\cdot) \in \mathcal{P}\left(\mathbb{T}^{n}\right)$. Let $0<r \leq 1, \alpha \in L^{p^{\prime}(\cdot)}$, and let $\sigma(\xi)$ be a symbol such that

$$
\sum_{\xi \in \mathbb{Z}^{n}}|\sigma(\xi)|^{r}<\infty
$$

Then $\alpha T_{\sigma}$ is $r$-nuclear from $L^{p(\cdot)}$ to $L^{q(\cdot)}$ for all $q(\cdot) \in \mathcal{P}\left(\mathbb{T}^{n}\right)$. If additionally $p^{+}<\infty, r \leq \frac{2}{3}$, and $q(\cdot)=p(\cdot)$, then $\alpha T_{\sigma}$ is $r$-nuclear in $\mathcal{L}\left(L^{p(\cdot)}\left(\mathbb{T}^{n}\right)\right)$ and

$$
\operatorname{Tr}\left(\alpha T_{\sigma}\right)=\int_{\mathbb{T}^{n}} \alpha(x) d x \cdot \sum_{\xi \in \mathbb{Z}^{n}} \sigma(\xi)=\sum_{j=1}^{\infty} \lambda_{j},
$$

where $\lambda_{j}(j=1,2, \ldots)$ are the eigenvalues of $\alpha T_{\sigma}$ with multiplicities taken into account. 
In particular, let us consider the symbol $\sigma(\xi)=\left(1+4 \pi^{2}|\xi|^{2}\right)^{-\frac{\tau}{2}}$ for $\tau>0$. The corresponding multiplication yields the operator $\alpha T_{\sigma} f=$ $\alpha(I-\Delta)^{-\frac{\tau}{2}} f$ on $\mathbb{T}^{n}$. We observe that $\sum_{\xi \in \mathbb{Z}^{n}}\left(1+4 \pi^{2}|\xi|^{2}\right)^{-\frac{r \tau}{2}}<\infty$ if and only if $r \tau>n$. Consequently we obtain:

Corollary 3.4. Let $p(\cdot) \in \mathcal{P}\left(\mathbb{T}^{n}\right)$. If $0<r \leq 1$, $\alpha \in L^{p^{\prime}(\cdot)}$, and $r \tau>n$, then $\alpha T_{\sigma}=\alpha(I-\Delta)^{-\frac{\tau}{2}}$ is r-nuclear from $L^{p(\cdot)}$ to $L^{q(\cdot)}$ for all $q(\cdot) \in \mathcal{P}\left(\mathbb{T}^{n}\right)$. If additionally $p^{+}<\infty, r \leq \frac{2}{3}$, and $q(\cdot)=p(\cdot)$, then $\alpha(I-\Delta)^{-\frac{\tau}{2}}$ is r-nuclear in $\mathcal{L}\left(L^{p(\cdot)}\left(\mathbb{T}^{n}\right)\right)$ and

$$
\operatorname{Tr}\left(\alpha(I-\Delta)^{-\frac{\tau}{2}}\right)=\int_{\mathbb{T}^{n}} \alpha(x) d x \cdot \sum_{\xi \in \mathbb{Z}^{n}}\left(1+4 \pi^{2}|\xi|^{2}\right)^{-\frac{\tau}{2}}=\sum_{j=1}^{\infty} \lambda_{j},
$$

where $\lambda_{j}(j=1,2, \ldots)$ are the eigenvalues of $\alpha(I-\Delta)^{-\frac{\tau}{2}}$ on $L^{p(\cdot)}\left(\mathbb{T}^{n}\right)$ with multiplicities taken into account.

\section{REFERENCES}

[ACPP05] G. Alberti, M. Csörnyei, A. Pełczyński, and D. Preiss. BV has the bounded approximation property. J. Geom. Anal., 15(1):1-7, 2005.

[CUFRW14] D. Cruz-Uribe, A. Fiorenza, M. Ruzhansky, and J. Wirth. Variable Lebesgue Spaces and Hyperbolic Systems, Advanced Courses in Mathematics - CRM Barcelona. Birkhäuser, Basel, 2014.

[DHHR11] L. Diening, P. Harjulehto, P. Hästö, and M. Růžička. Lebesgue and Sobolev spaces with variable exponents, Lecture Notes in Mathematics. Springer-Verlag, Heidelberg, 2011.

[DR] J. Delgado and M. Ruzhansky. The metric approximation property for variable exponent Lebesgue spaces and nuclearity. arXiv:1503.07202v1.

[DRW] J. Delgado, M. Ruzhansky, and B. Wang. Approximation property and nuclearity on mixed-norm $L^{p}$, modulation and Wiener Amalgam spaces. to appear in J. Lond. Math. Soc. arXiv:1410.4687v2.

[Enf73] P. Enflo. A counterexample to the approximation problem in Banach spaces. Acta Math., 130:309-317, 1973.

[FG89] H. G. Feichtinger and K. H. Gröchenig. Banach spaces related to integrable group representations and their atomic decompositions. II. Monatsh. Math., 108(2-3):129-148, 1989.

[Gro55] A. Grothendieck. Produits tensoriels topologiques et espaces nucléaires. Mem. Amer. Math. Soc., 1955(16):140, 1955.

[Iwa10] T. Iwabuchi. Navier-Stokes equations and nonlinear heat equations in modulation spaces with negative derivative indices. J. Differential Equations., 248(8):1972-2002, 2010. 
[JS12] W. B. Johnson and A. Szankowski. Hereditary approximation property. Ann. of Math. (2), 176(3):1987-2001, 2012.

[Lid59] V. B. Lidskiu. Non-selfadjoint operators with a trace. Dokl. Akad. Nauk SSSR, 125:485-487, 1959.

[LLO10] A. Lima, V. Lima, and E. Oja. Absolutely summing operators on $C[0,1]$ as a tree space and the bounded approximation property. $J$. Funct. Anal., 259(11):2886-2901, 2010.

[RSTT11] M. Ruzhansky, M. Sugimoto, J. Toft, and N. Tomita. Changes of variables in modulation and Wiener amalgam spaces. Math. Nachr., 284(16):2078-2092, 2011.

[RSW12] M. Ruzhansky, M. Sugimoto, and B. Wang. Modulation spaces and nonlinear evolution equations. In Evolution equations of hyperbolic and Schrödinger type, volume 301 of Progr. Math., pages 267-283. Birkhäuser/Springer Basel AG, Basel, 2012.

[RT10a] M. Ruzhansky and V. Turunen. Pseudo-differential operators and symmetries. Background analysis and advanced topics, volume 2 of Pseudo-Differential Operators. Theory and Applications. Birkhäuser Verlag, Basel, 2010.

[RT10b] M. Ruzhansky and V. Turunen. Quantization of pseudo-differential operators on the torus. J. Fourier Anal. Appl., 16(6):943-982, 2010.

[Sza81] A. Szankowski. $B(H)$ does not have the approximation property. Acta Math., 147(1-2):89-108, 1981.

Department of Mathematics, Imperial College London, 180 Queen's

Gate, London SW7 2AZ, United Kingdom

E-mail address: j.delgado@imperial.ac.uk

Department of Mathematics, Imperial College London, 180 Queen's Gate, London SW7 2AZ, United Kingdom

E-mail address: m.ruzhansky@imperial.ac.uk

LMam, School of Mathematical Sciences, Peking University, BeiJING 100871, CHINA

E-mail address: wbx@pku.edu.cn 